\title{
THE INFLUENCE OF PHYTOTHERAPY ON MACROPROLACTINOMA SIZE
}

\author{
Ivo Trogrlić*, Dragan Trogrlić, Zoran Trogrlić \\ "DREN" Ltd, Žepče, Bosnia \& Herzegovina \\ 1Bukovik 3, 72230 Žepče, B \& H \\ *E-mail: dragan.trogrlic@tel.net.ba
}

\begin{abstract}
The study aims at demonstrating the efficiency of phytotherapy in macroprolactinoma downsizing. The comparison of phytotherapeutic (PT) efficiency leant on medical records submitted by the patients prior to the PT launch, indicating the diagnosis established based on the outcome of the nuclear magnetic resonance imaging (NMRI) and/or computed tomography (CT) of the affected site and bringing the results of field-of-vision examination and hormonal status determination. The insight into the documentation in reference provided the information on tumour size and prolactin levels. The data in reference were compared against medical records submitted following phytotherapy completion. This study brings the results of a five-year investigation into the influence of phytotherapy on the size of macroprolactinomas. In patients responsive to this kind of treatment, tumour regression was witnessed within 6 months of herbal remedy use. A substantial tumour downsizing was accompanied by vision restitution; namely, in a number of cases, sight impairments are the first indicators of tumour presence. The basic parameters used for phytotherapeutic efficiency estimation and follow-up were the size of the tumour and the state of the field-ofvision, established both prior to and following phytotherapy. The results of the study presented herein unequivocally demonstrated the efficiency of phytotherapy in macroprolactinoma tumour mass downsizing, providing therefore solid grounds for the implementation of phytotherapy as a novel treatment modality of this tumour cluster.
\end{abstract}

Key words: Phytotherapy, macroprolactinoma, visual field defect

\section{Introduction}

Prolactinomas are the most common functional tumours of the pituitary gland, representing an approximate 45-percent share of the total hormone-releasing tumour pool (Horvath and Kovacs, 1992). Nearly $90 \%$ of prolactinomas are of the lactotrophic origin, while the remaining $10 \%$ are mixed tumours built up of both lactotrophic and somatotrophic cells characterised by enhanced prolactin and growth factor release (Scheithauer et al, 1986). Prolactinomas mutually differ in their size and secretion activity. Based on their size, they can be divided into microand macroprolactinomas. Microprolactiomas grow slowly, up to only a few millimetres in size and are usually distinctive and not prone to grow further (Mah and Webster, 2001; Guyton and Hall, 2003). In the majority of the diseased, macroprolactinomas grow over $10 \mathrm{~mm}$ in size; their growth is mostly rapid and expansive so that they infiltrate the adjacent anatomic structures and pressurise them, endangering thereby health and even life of the diseased (Mah and Webster, 2001). Of a particular note, the fact that the pituitary gland is seated next to the optical structures enables macroprolactinomas to pressurise the optical nerve and/or the optical chiasm, so that some patients may experience more or less pronounced visual field defects. The latter often represent the first symptom of a macroprolactinoma presence.

As compared to macroprolactinomas, the secretion activity of microprolactinomas is by far lower. Due to the high level of prolactin they synthesise and release, macroprolactinomas are featured by a distinctive clinical presentation (Asa, 1988; Levy and Lightman, 1993). In order to treat them, contemporary medicine mostly resorts to dopamine agonists (DAs) as the first-line pharmacotherapy. However, microprolactinomas and macroprolactinomas differ in their sensitivity to such a treatment (Braucks et al, 2003). While microprolactinomas are only poorly responsive, a substantial reduction in prolactin level, but not in tumour size, can be seen in most patients diagnosed with macroprolactinomas and taking Das. (Levy and Lightman, 1993). On top of dopamine agonists, contemporary medicine makes use of microsurgery and radiotherapy (Horvath and Kovacs, 1992, Braucks et al, 2003).

This study aims at demonstrating the efficiency of phytotherapy in macroprolactinoma downsizing and strives to prove that pharmacoactive ingredients present in remedial plant mixtures are ultimately capable of attaining tumour regression. 


\section{Subjects and Methods}

The study was carried out within 2005 - 2010 timeframe and comprised a total of 32 patients, out of which 21 (65.6\%) women and 11 (34.4\%) men, all of them being the citizens of currently independent states occupying the territory of the former Yugoslavia (Bosnia and Herzegovina, Croatia, Serbia and Macedonia). The patients' age spanned from 16 to 78 (average age, 40.7). Prior to PT commencement, the patients submitted medical records revealing their diagnosis established based on the nuclear magnetic resonance imaging (NMRI) and/or computed tomography (CT) of the affected site, hormonal status and field-of-vision examination. These data served as the baseline for comparison and follow-up of the PT efficiency.

Throughout the study course, macroprolactinoma patients had been treated with three distinctive plant remedies (Preparations 1, 2 \& 3). These preparations mutually differ in their composition and consist solely of pounded herbs lacking any additives whatsoever. The constitutive plants are pounded to the standard degree (Lukić, 1993). Flowers, stalks and leaves were prepared using a number 6 sieve (rough cutting), roots and barks using a number 3 sieve, and seeds and fruits using a number 2 sieve (precise cutting) (Pekić, 1983; Kovačević, 2000). These plant preparations are prepared and drunk in form of tea and have to be used on an everyday basis in line with the following schedule: Preparation 1 at 7:00 am, Preparation 2 at 2:00 pm, and Preparation 3 at 7:00 pm. All of them are prepared in the same way, a single tea dose thereby calling for $1.5 \mathrm{~g}$ of the herbal mixture and $200 \mathrm{~cm}^{3}$ of water.

Following the analysis of the PT efficiency in terms of tumour mass downsizing carried out on the whole study sample level, the subjects were divided into two groups.

Group 1 (hereinafter referred to as: the PT only arm) consisted of 9 patients receiving only PT. Out of them, $8(88.9 \%)$ were women and $1(11.1 \%)$ was a man. They were aged 37.5 on the average (range, 20-70). Group 2 (hereinafter referred to as: the DA \& PT arm) consisted of 23 patients, in specific, 10 men (43.47\%) and 13 women $(56.53 \%)$ receiving PT in addition to their regular DA medication. The group in reference was aged 41.9 on the average (range, 16-78).

Our next goal was to determine the manner in which the length of previous DA therapy affects PT outcomes. To that end, the members of the DA \& PT arm were further subdivided into two subgroups based on the length of their pre-PT DA therapy. Subgroup 2A embraced the patients previously treated with DAs for 8-26 months and consisted of 19 patients, out of which $12(63.1 \%)$ were women and 7 (36.9\%) were men. The patients were aged 41.4 on the average, the youngest among them being 16 and the oldest one being 78. Prior to the PT launch, 4 of our study patients had been treated with DAs for over 36 months (Subgroup 2B). This subgroup embraced 3 (75\%) men and one (25\%) woman at the average age of 44.7 (range, 22-59).

Out of the entire study pool, 15 (46.8\%) patients had experienced more or less severe pre-PT visual symptoms arising on the grounds of compression of optical structures exerted by macroprolactinoma. Out of those, 8 $(53.3 \%)$ were of a female and $7(46.7 \%)$ of a male gender. These patients were aged 40 on the average (range, 18-59). Within the frame of the statistical analysis and outcome reporting, this patient subgroup was processed and presented separately.

The comparison between the pre-PT and post-PT health statuses carried out throughout the follow-up period made use of the following key endpoint measures:

1. Data on tumour size;

2. Data on the state of the field-of-vision of the patients suffering from pre-PT visual field defects;

3. Data on prolactin levels;

4. Data on prior treatments;

5. Data on PT length and side-effects.

The obtained data served as the basis for mathematical-statistical analysis aiming at corroborating or refuting certain PT administration-related hypotheses. In order to obtain as reliable conclusions as possible, the credibility of the obtained data was analysed first; in further course, the analysis of data on tumour size prior to and following PT was carried out in order to verify the PT efficiency. In pursue of this path, the hypothesis on the significance of differences in pre-PT and post-PT tumour dimensions was tested at 5\%-, 1\%-, and $0.1 \%$-significance levels; the significance threshold of $>5 \%$ was used only in cases of a scarce relevant information.

In addition to the standard statistics, the testing of significance of the differences between the endpoint measures obtained prior to and following phytotherapy employed the following non-parametric statistics: the paired sample sign test, Wilcoxon sign-rank test, Mann-Whitney U-test and McNemar's test.

The statistical analysis was run through the following software:

1. IBM SPSS Statistics 13.0 for Windows

2. DATA ANALYSIS USING Microsoft EXCEL

The results of the hypothesis accuracy testing shall be given not only in form of figures, but in form of graphs as well. 


\section{Results}

As pointed out previously, data on the efficiency of the pituitary gland tumour phytotherapy (PT) came as a result of a five-year follow-up of relevant endpoint measures obtained across the sample of 32 macroprolactinoma patients. In the pertaining diagrams and tables, the patients were assigned ordinal numbers spanning from 1 to 32 , their sequence thereby matching the order of their applications. Within this particular study frame, the efficiency analyses were focused on changes in tumour size.

\section{Analysis of changes in tumour size witnessed following PT as compared to the pre-PT status - the entire patient sample}

The basic tumour size indicators obtained by virtue of descriptive statistics revealed significant changes in the mean tumour size prior to and following PT.

Prior to PT, the mean tumour size equalled to $22.00 \mathrm{~mm}$; following PT, it decreased to $9.72 \mathrm{~mm}$. This is to say that a $56 \%$-tumour downsizing was achieved. Based on the latter result, the assumed PT efficiency in tumour downsizing was corroborated at the level of significance of $1 \%$. The same was obtained using the $0.1 \%$-significance merit. Graphical display of the results descriptive of differences in tumour sizes prior to and following PT, obtained on the entire sample level, is given in Diagram 1.

\section{Group 1: PT only arm}

Following the analysis of changes in tumour size witnessed across the entire study sample, the patients were divided into 2 groups; in further course, the analyses of changes in tumour size were separately run across the PT only (Group 1) and the DA \& PT arm (Group 2).

Out of 32 participating patients, 9 gave up on DAs prior to PT and continued taking herbal remedies only (PT only arm). The basic parameters descriptive of the tumour size, obtained by virtue of descriptive statistics, revealed a significant difference in the mean tumour size prior to and following PT. Prior to PT, the mean tumour size equalled to $20.11 \mathrm{~mm}$; following PT, the mean tumour size decreased to $3.4 \mathrm{~mm}$, meaning that an $83 \%$-tumour downsizing ultimately managed to be attained (Diagram 2).

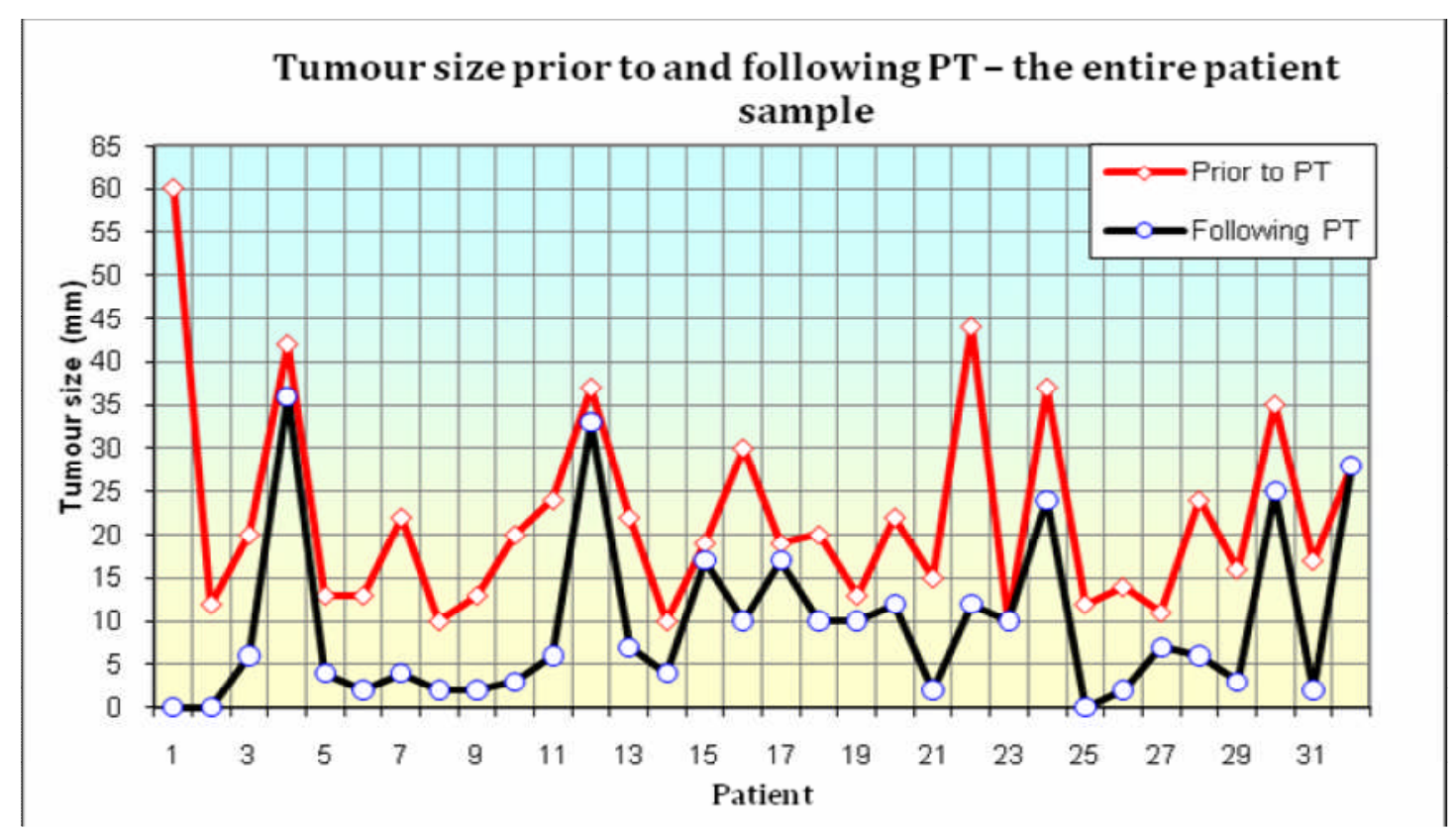

Diagram 1

Note: During the course of PT female patients number 19 and 23 managed to conceive due to the decrease in prolactin levels, so the data on their tumour size are lacking. Therefore, these patients were excluded from the statistical analysis. For the sake of further data processing, the sizes of their tumours were deemed to be equal to the mean tumour size seen across their respective study groups (see Diagrams 1,3 and 4).

The statistical significance of the tumour mass downsizing seen following PT, as compared to the pre-PT state, was corroborated at the level of significance of $5 \%$. 


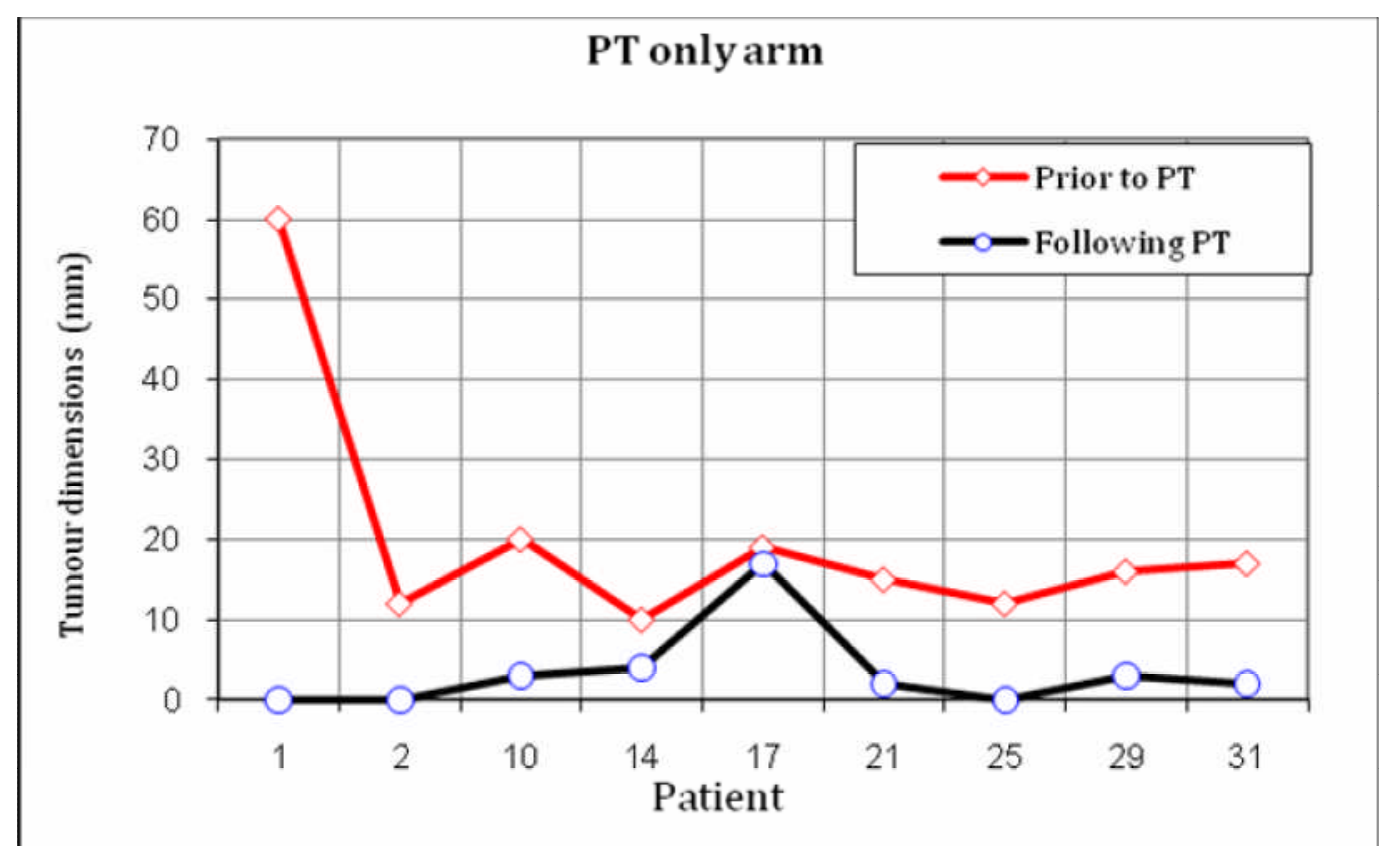

Diagram 2

However, if tested at the significance level of $1 \%$, the difference in pre- and post-PT tumour sizes became statistically insignificant.

\section{Group 2: DA \& PT arm}

DA \& PT arm was constituted of 23 patients simultaneously receiving phytotherapy and dopamine agonists. In this patient group, the basic parameters descriptive of tumour size, obtained by virtue of descriptive statistics, also revealed a significant difference in the mean tumour size prior to and following PT. The mean pre- and post-PT tumour sizes registered across these patients were $22.74 \mathrm{~mm}$ and $11.93 \mathrm{~mm}$, respectively (Diagram 3); in other words, a 48\%-tumour downsizing was achieved. The statistical significance of the difference in tumour size prior to and post the combined treatment was corroborated both at the level of significance of $1 \%$ and at the level of significance of $0.1 \%$.

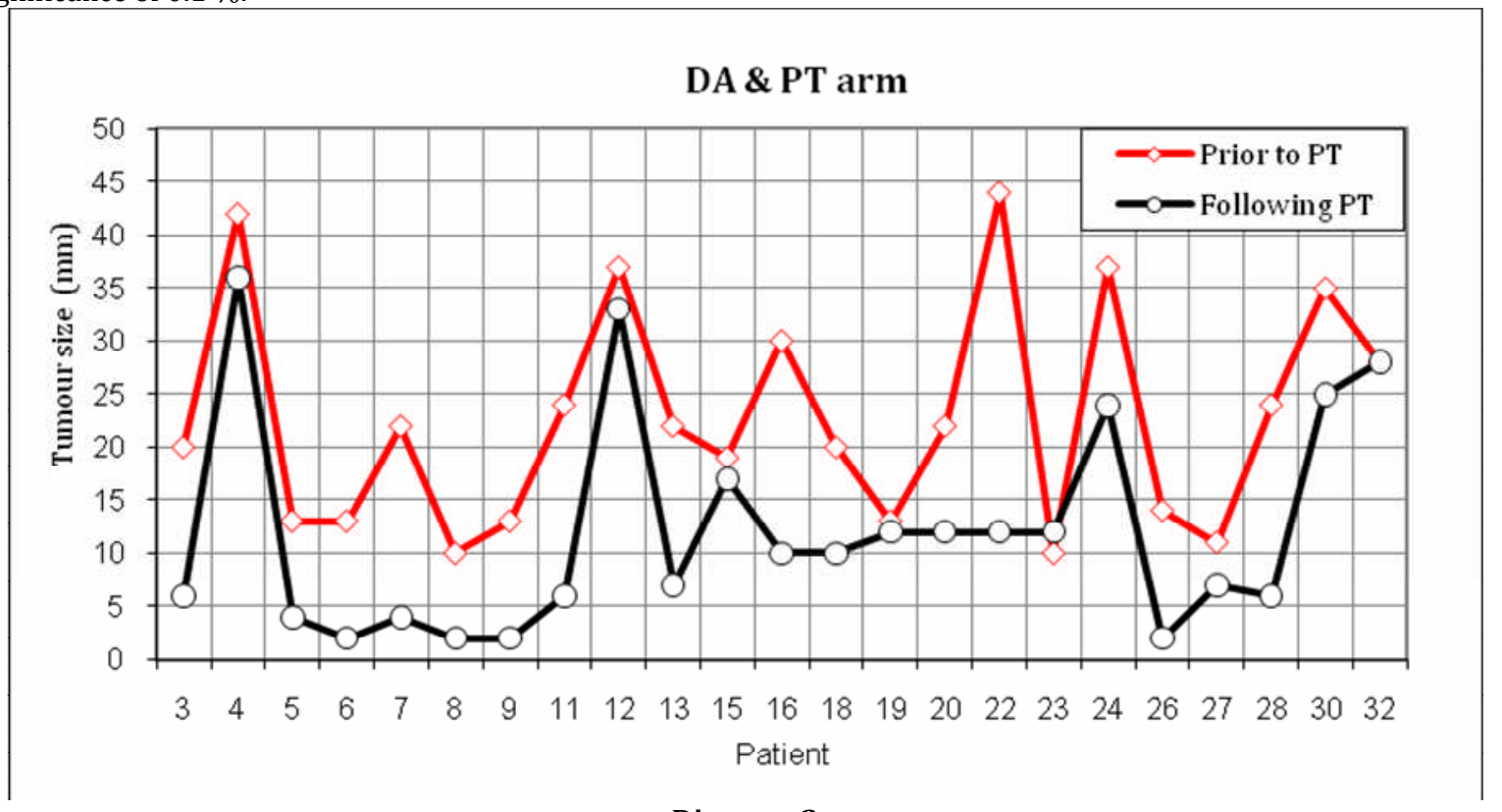

Diagram 3 
The comparison of tumour downsizing attained in Group 1 vs Group 2

Based on the data on mean tumour sizes and other statistical indicators, a comparative analysis of data on tumour sizes registered in PT only and DA \& PT arms was carried out. This analysis aimed at comparing the outcomes of the two different treatments. When tested for their individual significance at 5\%-significance level, the differences in mean tumour sizes were proven to be insignificant. Comparative analysis of mean tumour sizes registered following PT only and following the concomitant DA and PT treatment confirmed the anticipated efficiency of both treatment modalities. The testing ran at the level of significance of 5\% revealed the phytomonotherapy, as well as DA \& PT combination therapy, to be capable of statistically significantly reducing the size of the tumour, which can justifiably be attributed to PT administration. Nevertheless, the same failed to be proven at the significance level of $1 \%$, suggesting that a more reliable comparative analysis would call for larger patient samples.

\section{Subgroup 2A - DA \& PT arm: Patients previously receiving DAs for 8 - 26 months}

The next step taken during the course of the study was to verify the impact of previous DA treatment on PT outcomes. To that goal, the total of 23 DA \& PT patients was divided into two subgroups: Subgroup 2A, consisting of 19 patients previously receiving DAs for 8 to 26 months, and Subgroup 2B, consisting of 4 patients previously receiving DAs longer than 36 months.

In Subgroup 2A, whose members had received DAs for 8 to 26 months prior to the PT launch, statistically significant differences in mean tumour sizes prior to and following the combined therapy were revealed. Prior to the PT commencement, the mean tumour size seen across these patients equalled to $19.9 \mathrm{~mm}$, as compared to $8.9 \mathrm{~mm}$ measured following the PT completion, indicating a 56\%-downsizing of the tumour mass. The difference in pre-PT and post-PT mean tumour sizes remained significant at 5\%-, 1\%-, and 0.1\%-significance levels. The results are graphically represented by Diagram 4.

\section{Subgroup 2B: DA \& PT arm: Patients previously receiving DAs > 36 months}

Prior to the PT commencement, 4 of the study subjects had taken DAs for longer than 36 months. Prior to the PT implementation, the mean size of the tumour equalled to $36.0 \mathrm{~mm}$; following the combined DA \& PT therapy, the mean size of the tumour was reduced to $26.0 \mathrm{~mm}$ (Diagram 5), hence by $28 \%$. Statistical indicators corroborated

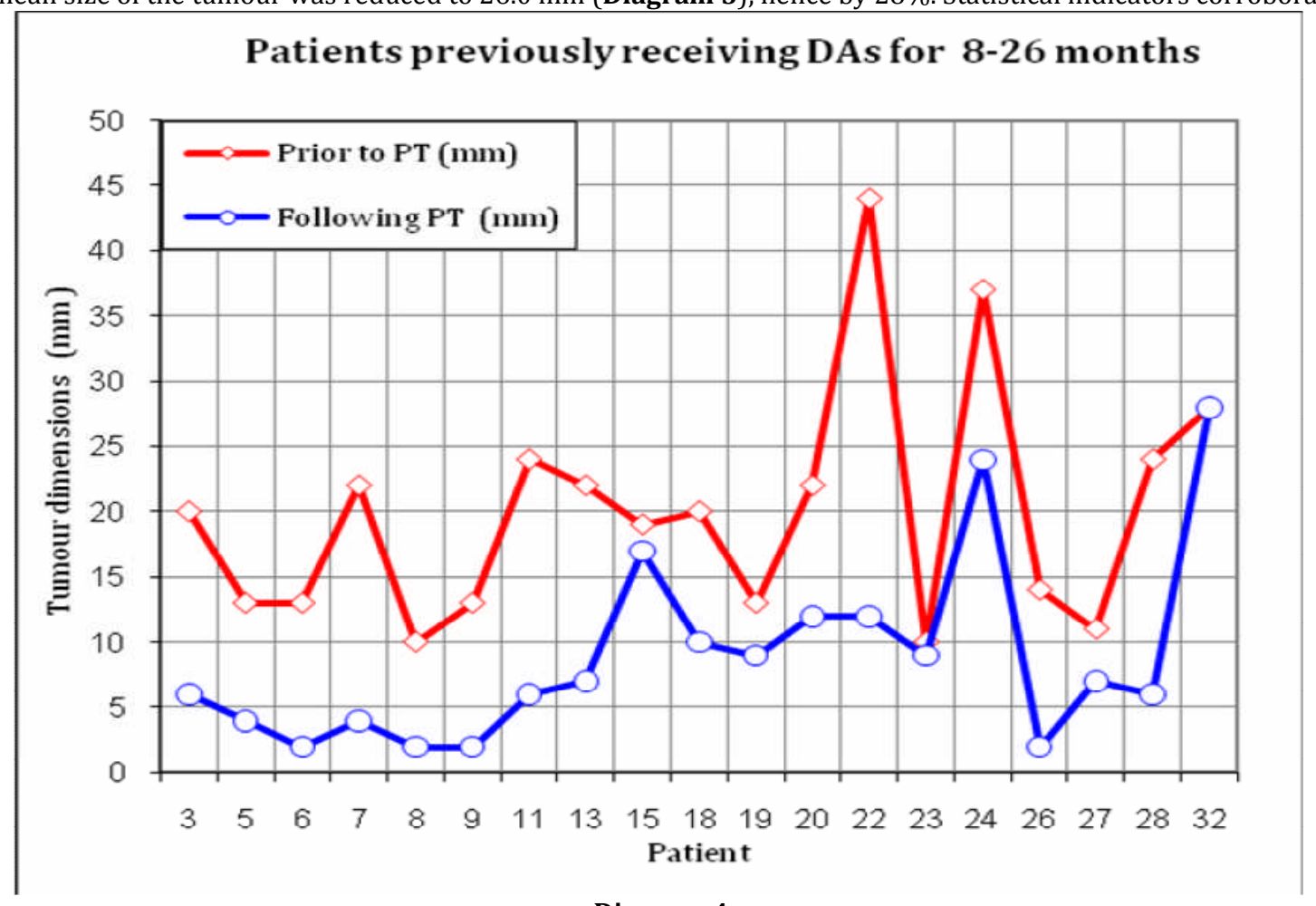

Diagram 4 


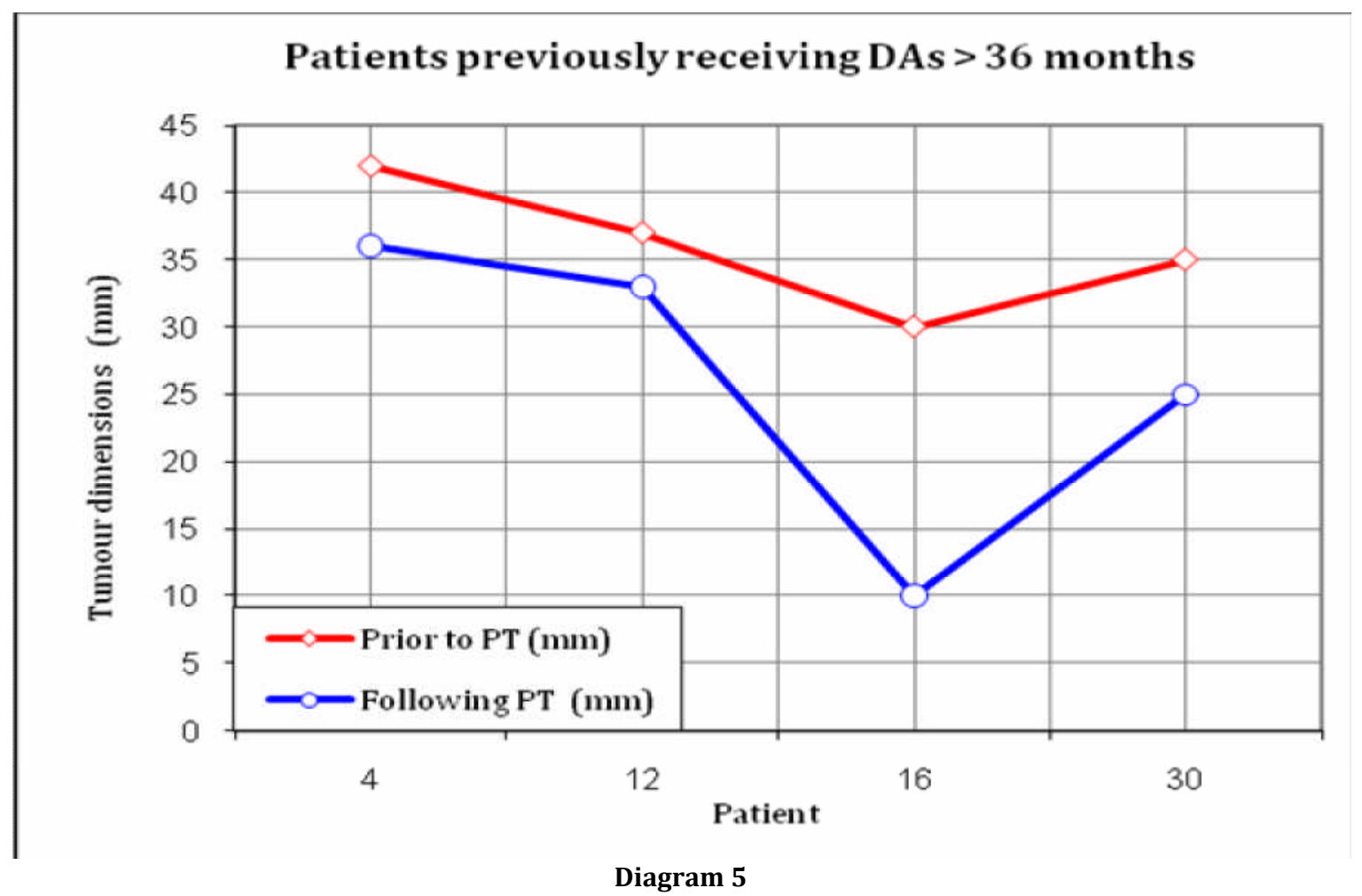

that certain changes in tumour size did occur, but the difference in tumour sizes prior to and following the combined therapy lacked statistical significance, at least at the level of significance of $5 \%$. The results obtained in this patient subgroup might be indicative of an unfavourable impact of a multiyear DA therapy reflecting in the PT efficiency hindrance. However, given the fact that the subgroup in reference was composed of no more than 4 patients, one should not jump to conclusions, but rather provide for a more representative future patient sample. Nevertheless, it is to be reiterated that, even though insignificant, certain tumour regression was noted in these cases as well.

\section{visual field defect (VFD) and the size of the tumour}

VFD comes as a result of tumour compression exerted on optical structures. The results displayed below prove the favourable impact of phytotherapy on tumour downsizing.

The impact of PT on VFD was not analysed in all of the 32 patients, but only in those 15 that had experienced vision problems prior to the PT commencement (Table 1). 
Table 1 VFD status prior to and following PT

\begin{tabular}{|c|c|c|}
\hline $\begin{array}{c}\text { Patient's ordinal } \\
\text { number }\end{array}$ & VFD prior to PT & $\begin{array}{c}\text { VFD following } \\
\text { PT }\end{array}$ \\
\hline 1 & + & - \\
\hline 3 & + & - \\
\hline 4 & + & $+^{*}$ \\
\hline 10 & + & - \\
\hline 11 & + & - \\
\hline 12 & + & $+^{*}$ \\
\hline 16 & + & - \\
\hline 17 & + & + \\
\hline 20 & + & - \\
\hline 22 & + & - \\
\hline 24 & + & + \\
\hline 26 & + & - \\
\hline 29 & + & - \\
\hline 30 & + & + \\
\hline 32 & + & + \\
\hline \multicolumn{3}{|l|}{ Abbreviations: } \\
\hline \multicolumn{3}{|l|}{ PT phytotherapy } \\
\hline \multicolumn{3}{|c|}{ VFD visual field defect } \\
\hline \multicolumn{3}{|l|}{$(+)$ VFD present } \\
\hline \multicolumn{3}{|l|}{ ( - ) VFD lacking } \\
\hline$+^{*}$ permanent VF & & \\
\hline
\end{tabular}

Following PT, in $9(60 \%)$ out of these 15 patients, the vision was restored to normal. In these patients, the reduction in tumour size seen following PT spanned from 45\% to 100\%. In 6 (40\%) patients in whom a VFD persisted despite of PT, the attained tumour downsizing ranged from $0 \%$ to 35\% (marked with black dots in Diagram 6). As can be seen from the data, in patients lacking a VFD following PT, the reduction in tumour size was far greater than in those having a persistent, PT-irresponsive VFD (statistical significance of the difference in tumour downsizing proven at the level of significance of $5 \%$, but not at the level of significance of $1 \%$ ).

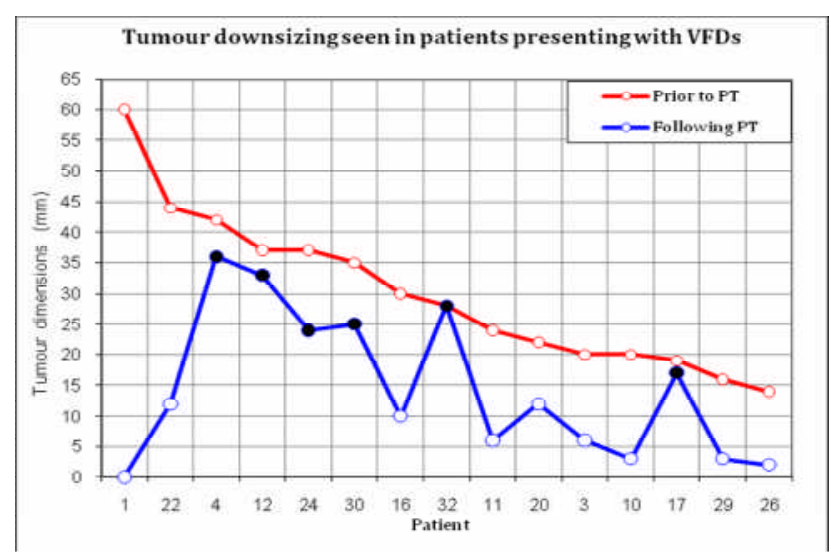

Diagram 6 
Statistical analyses proved a close correlation between the tumour downsizing and the VFD status. Duly chosen tests examining the interrelation between these two variables ran at the level of significance of $0.1 \%$, definitely proved their interdependence.

\section{Discussion}

In addition to more or less striking side-effects, among them a higher risk of developing heart fibrosis serving the EMEA as the grounds for limiting the maximal permissible daily DA doses (EMEA; Europe, 2011), DA treatment also leads to changes in tumour structure. In a number of bromocriptine-treated patients, intra-tumour bleedings and cyst formation have been seen. All DAs, bromocriptine in particular, enhance tumour fibrosis, which hinders surgery and narrows its scope to a substantial degree (Pehaček and Sajdl, 1990). Given the fact that previous long-term DA treatment diminished PT efficiency in our patients as well, it is safe to say that, when considering treatment options, PT should be chosen over DAs. The fact that patients undergoing PT for 2-41 months failed to experience any side-effects whatsoever throughout the PT course regardless of its length further supports the above position statement. The fact that two female subjects (allocated ordinal numbers 19 and 23) got pregnant while on PT also speaks in favour of PT safety. Both of them were originally assigned to the DA \& PT arm, but decided to quit DAs once they got pregnant and stuck to PT, which went smoothly throughout the entire pregnancy. Following the DA cessation, PT was recommended to be continued due to pregnancy-related hyperestrogenism and threatening oestrogen-enhanced tumour growth (Mah and Webster, 2001).

In patients responding to PT, tumour downsizing was witnessed within 6 months of the PT commencement, the best results thereby being obtained in patients continuously taking herbal remedies for 10 or more months.

\section{The impact of PT on macroprolactinoma size}

The subjects included into this study had unanimously started their macroprolactinoma treatments with the most commonly employed dopamine agonist bromocriptine. Nowadays, medical research is designed so as to provide the intention-to-treat population with the "gold standard" therapy for the condition under investigation (Geddes and Cipriani, 2004). Respecting that principle, our DA-treated patients also continued taking DAs concurrently with PT. Mono-PT was administered only to patients forced to give up on DAs due to severe side-effects. Any other approach to this study would jeopardise its ethics and integrity. Nevertheless, this line of approach had a substantial impact on our study design and resulted in the formation of several study groups/subgroups. Based on the aforementioned, the researchers were given the opportunity not only to observe the impact of PT on tumour size across the entire (32-) patient sample, but to compare the results obtained with PT only (9-patient) arm and DA \& PT (23-patient) arm.

On top of medication, a total of 15 study patients had surgery prior to the PT commencement. The surgery allowed for the removal of almost the entire tumour mass, as well as for the subsequent depressurisation of the adjacent structures and temporary alleviation of macroprolactinoma-related symptoms. However, none of the patients had his/her tumour totally removed, so that in time the residual tumour cells served as the grounds for macroprolactinoma re-formation. In four of the operated patients, the surgery failed even twice. Medical records obtained prior to the PT commencement clearly showed that each and every study subject had an optimal treatment delivered by their attending healthcare facilities, so that, as far as contemporary medicine is concerned, the best possible care had been provided.

The herbal remedy efficiency analysis carried out on the entire sample level and particularly the results obtained across the PT only arm have clearly demonstrated the favourable impact of herbal remedies on tumour mass reduction. The results attained across the PT only arm represent the most important outcome of this study, since they showed beyond any doubt that tumour mass reduction and even restoration to health (patients Nos 1,2 , and 25) can be achieved in those suffering from macroprolactinomas solely by virtue of herbal remedy use, allowing for the possibility of PT implementation as the first-line macroprolactinoma treatment. A substantial, however less striking effect in terms of tumour mass reduction was also achieved in the DA \& PT arm. Given that the statistical analysis revealed no significant pre-PT differences between the mean sizes of the tumours seen in patients who gave up on their medication as compared to those still taking DAs, it became obvious that pharmacotherapy had no significant impact on tumour size, so that the substantial tumour downsizing can mostly be attributed to PT implementation.

This is the very first time that a study actually managed to firmly prove the efficiency of herbal remedies in macroprolactinoma downsizing; namely, at least to our knowledge, similar studies have never been published before.

\section{Impact of the previous DA treatment on the PT outcome}

Far better results obtained with the PT only arm (average tumour downsizing of 83\%) as compared to the DA \& PT arm (average tumour downsizing of 48\%), suggest that, when it comes to tumour mass reduction, earlier DA treatment might decrease the PT efficiency. Further analysis of the results obtained in DA-treated patients (Subgroups $2 \mathrm{~A}$ and $2 \mathrm{~B}$ ) led one to conclude that therapeutic effectiveness of herbal remedies weakens as DA administration goes 
on, so that the poorest results were obtained in patients treated with DAs for longer than 3 years; the average tumour mass reduction seen across these patients (56\% in Subgroup 2A and 28\% in Subgroup 2B) supports this standing. The analysis of the study data indicated that prior DA treatment interferes with signalling paths utilised by pharmacoactive plant ingredients, so that the PT efficiency declines with DA treatment duration. Based on the aforementioned, it can also be clearly understood that tumour downsizing, coming as a result of the pharmacoactive plant ingredients' administration, is not mediated by D2 receptors.

This article brings the report on favourable effects of herbal preparation administration. Nevertheless, the identification of individual pharmacologically active components is associated with a number of problems. The majority of plants composing the herbal preparations administered to macroprolactinoma patients are aromatic plants basically composed of ethereal oils; it has been shown without a shred of doubt that the pharmacoactivity of these oils gives rise to PT efficiency. However, given that these herbal preparations come in form of infusions, one should take into account that only some of their hydrophobic ingredients get to pass into the water solution, together with a number of other secondary metabolites. In line with the foregoing, it is reasonable to assume that pharmacological activity of aromatic plants does not depend solely on the presence and efficiency of ethereal oils, but also on the activity of other ingredients constituting the plant mixtures; for that matter, the possibility of their synergistic effect can not be ruled out.

One can also expect to come across a number of difficulties when trying to identify active ingredients of ethereal oils, since the activity of the latter actually represents a "combined effort" of all ingredients. Therefore, the pharmacoactivity of aromatic plants differs from that of ethereal oils isolated from such plants (Kovačević, 2000). Due to the aforementioned, the pharmacological activity of ethereal oils is commonly defined for the oil "in toto" rather than for its individual components whose pharmacokinetics and metabolism are often very hard or virtually impossible to track. Consequently, most studies akin to our actually report on the effects of PT administration and make ex iuvantibus judgements.

Elucidation of the exact action mechanism through which active substances present in herbal mixtures used within this study take their course of action and eventually manage to downsize the tumour shall contribute to better understanding of the development of tumours of neurohormonal aetiology.

\section{PT impact on visual field defect (VFD)}

In 15 study participants, the size of the tumour and the direction of its growth finally caused a visual field defect (VFD). The subjects had been subjected to occasional MRI or CT controls, most often scheduled every 6 months; in those who experienced tumour regression, clearance of sight symptoms posed as the first sign of tumour downsizing, later corroborated by virtue of the pituitary gland diagnostic imaging. In addition to optical structures' depressurisation, a smaller degree of sinus involvement was established in patients having their tumours penetrated into the cavernous sinuses. The macroprolactinoma invasion into the cavernous sinuses speaks of its aggressiveness, forecasts a poor prognosis and makes the tumour virtually inoperable (Asa, 1988). In patients whose tumours became substantially smaller, full cavernous sinus riddance from the tumour was noted, making the chances for a successful surgery markedly higher. In these patients, vision recovery took place within 6 months of the PT commencement. Of note, even prior to PT, two of our patients had been diagnosed with a permanent visual field defect, so that much could not be done anyway.

The results brought by this article demonstrate the efficiency of PT in macroprolactinoma downsizing, therefore opening the prospects for the following:

- $\quad$ Non-surgical treatment and consequential avoidance of risks associated with surgical procedures;

- $\quad$ Adjuvant therapy, to be administered to those whose tumour is inoperable either due to its size or due to the position of its seat, as well as to those who refuse to undergo surgery or can not have it due to contraindications;

- $\quad$ Administration of phytotherapy to the effect of preoperative tumour downsizing, allowing for the possibility of a more effective subsequent surgery;

- $\quad$ The possibility of a preventative PT, to be carried out in pregnant women suffering from macroprolactinoma and refusing DAs during pregnancy.

\section{Conclusion}

Our previous study (Trogrlić et al, awaiting publication) showed the usefulness of PT in macroprolactionoma secretion activity control. The results of this study demonstrated its additional efficiency in tumour downsizing. The outcomes seen across patients whose tumours became virtually undetectable following PT clearly demonstrate that some of the patients might be restored to health solely by virtue of phytotherapy administration. 


\section{References}

1. Asa, S.L. (1988). Tumours of the Pituitary Gland. In: Rosai, J., Sobin, H. Armed Forces Institute of Pathology, Washington D.C. , third series, Fasc $22: 1-120$.

2. Braucks, G.R., Naliato, E.C., Tabet, A.L., Gadelha, M.R. and Violante, A.H. (2003). Clinical and therapeutic aspects of prolactinoma in men. Arq. Neuropsiquiatr., 61: 1004-1110.

3. Geddes, J.R. and Cipriani, A. (2004). Selective serotonin reuptake inhibitors. BMJ; 329:809-10.

4. Guyton, A.C. and Hall, J. E. (2003). Textbook of Medical Physiology, Philadelphia, Pennsylvania : W.B.

Saunders Company : 846 - 857

5. Horvath, E. and Kovacs, K. (1992). Ultrastructural diagnosis of human pituitary adenomas. Microsc. Rec. Tech., 20: 107- 135.

6. $\quad$ http://www.emea.europa.eu/pdfs/human/press/pr/32239508en.pdf

7. Kovačević, N. (2000). Pharmacognostic basics. Faculty of Pharmacy University of Belgrade: 35-212.

8. Levy, A and Lightman, S.L. (1993). The pathogenesis of pituitary adenomas. Clinical Endocrinology; $38: 559$

9. Lukić, P.B. (1993). Pharmacognostics. Faculty of Pharmacy University of Belgrade: 18 - 24.

10. Mah, P.M. and Webster, J. (2001). Hyperprolactinaemia: aetiology, diagnosis and management. Semin. Reprod. Med., 20: 365-374.

11. Pehaček, Z. and Sajdl, P. (1990). Ergot alkaloids: Chemistry, biological effects, biotechnology. Prague Academy: 1-42.

12. Pekić, B. (1983). Chemistry and technology of pharmaceutical products - alkaloids and ether oils. Faculty of Technology University of Novi Sad: 1-68.

13. Scheithauer, B.W., Horvath, E., Kovacs K., Laws, E.R.Jr., Randall, R.V., Ryan, N. (1986). Plurihormonal pituitary adenomas. Semin Diagn Pathol; 3:69-82.

14. Trogrlić, I., Trogrlić, D., and Trogrlić, Z. The influence of phytotherapy on prolactin level in macroprolactinoma patients. Foreseen to be published in: AJTCAM 2012; 9 (1): 67-72. 